\title{
Simulations of Various Applications of Fuzzy Logic using the MATLAB
}

\author{
Dilpreet Kaur Grover \\ Final Year Student \\ Department of ECE \\ SDITS Khandwa (M.P.), India
}

\begin{abstract}
With the increasing problems of uncertainty, vagueness and imprecision during the modeling of various control system, the fuzzy logic comes into account. Fuzzy logic was first introduced by a Polish philosopher, Jan Lukasiewicz, in 1930. As the classical logic operates with only two values- 1 (true) and 0 (false), Lukasiewicz introduced a new logic which has more than two truth values. Lukasiewicz extends the range of classical logic to all the real numbers in the interval between 0 and 1 and named it as fuzzy logic. Fuzzy logic is a powerful tool which represents and process human knowledge in the form of fuzzy if-then rules. As the fuzzy logic systems is based on human thinking and natural language and also has a good stability, fast response and less complexity, the applications based on fuzzy logic have been increased significantly. To follow the trend, this paper presents the basic introduction of fuzzy logic, fuzzy sets and its operations. This paper provides a huge description of fuzzy logic system and fuzzy inference system and also provides comparison between fuzzy logic system and conventional control system. In this paper, the various applications of fuzzy logic have been simulated using the MATLAB.
\end{abstract}

\section{General Terms}

Boolean logic, image processing, signal to noise ratio.

\section{Keywords}

FIS, fuzzy logic, fuzzy rules, membership function.

\section{INTRODUCTION}

The control systems are generally explained by mathematical models which follow the stochastic models, law of physics or mathematical logic models. The trouble with such models is how to solve the given problem to a suitable mathematical model. No doubt, all such problems are overcome by today's advanced computer technology, but these systems are still too complex to manage. Hence, to simplify the uncertainty, vagueness and imprecision during the modeling, the concept of fuzzy logic comes into account.

In recent years, the applications of fuzzy logic have been increased significantly. Fuzzy logic can deal with information that is arising from computational perception, realization and cognition. In computing problems, it provides inclusion of obscure human evaluation. It also provides an impressive means for multiple criteria conflict resolution and preferable evaluation options. From certain and rough information, it addresses the solutions to that precise level as the decision is made by human, while the other approaches need definite and accurate equations to model the behaviors of real world.

Thousands of researchers and engineers are producing patents and research papers on fuzzy logic. Lotfi A. Zadeh proposed fuzzy logic first by his fuzzy set theory in 1965. According to Zadeh's report of March 4, 2013, the fuzzy logic made a huge impact on researchers. After that the research contributions rate is growing daily. Fuzzy logic is extremely useful for number of people involved in biomedical, agricultural, mechanical, electrical, computer software etc. for development and research [2].

\section{WHAT IS FUZZY LOGIC?}

Fuzzy logic concept is easy to understand. It is a form of multiple-valued logic which has more than two truth values. It uses the concept of degree of membership. In Boolean logic, the truth values may be only 0 or 1 , but in fuzzy logic, they will be any real number between 0 and 1 i.e. the truth values will range between true and false [4]. In mathematics, variables usually take numerical values, but in fuzzy logic applications, non-numeric values i.e. characters are used to eased forward the rules and to facts expressions. Fuzzy logic has two different meanings [1]:

- In narrow sense, fuzzy logic is an extension of multivalued logic.

- In broad sense, fuzzy logic is almost synonymous with the fuzzy set theory.

Fuzzy logic proposed various methods for deducing uncertain, incomplete and inaccurate knowledge. It compacts with reasoning which is inaccurate or imperfect rather than fixed, accurate and precise. The advantages of fuzzy logic are as follows [3]:

- Flexible, intuitive rule base design.

- Based on natural language, hence easy to use.

- Model non-linear function of licentious complexity.

- Simple implementation and easy computations.

- Multiple input signals can be handled easily.

- Increase robustness.

\section{FUZZY SETS}

Fuzzy set is a set that allows its members to have various degrees of membership within 0 and 1 i.e. within true and false. In other words, a set without crisp is a fuzzy set. A fuzzy set contains elements which have partial grades of membership [5].

Crisp values are those values which do not have clearly defined boundaries - for example, much longer than, tall, many, younger, etc. are true only to a certain degree as well as false to a certain degree. By contrast, fuzzy values are the values which have sharply defined values. Hence fuzzy sets do not contain any uncertain or incomprehensive values. 
In classical set theory, any given element can be completely excludes or completely includes i.e. an element either belong or does not belong to the set. While in fuzzy set theory, it allows gradual membership assessment of elements in a set which is explained with the membership function valued aid in the real unit interval $[0,1]$.

Commonly used example for fuzzy set theory is the set of tall people. The curve shown in fig: 1 is designated by $\mu$ and is known as a membership function. This curve varies from not tall to tall. If the set of people has a well defined (sharp edged) boundary, then the set is known as classical set. Figure 1 shows sharp-edged (non-fuzzy) membership function for tall. If the sharp defined boundary for tall people is 6 feet, then people have height more than 6 feet are considered tall. But in real people, if one person height is compared with another person, continuous membership function is used. Figure 2 shows continuous-edged (fuzzy) membership function for tall. The curve formed by this membership function varies smoothly between not tall and tall [1].

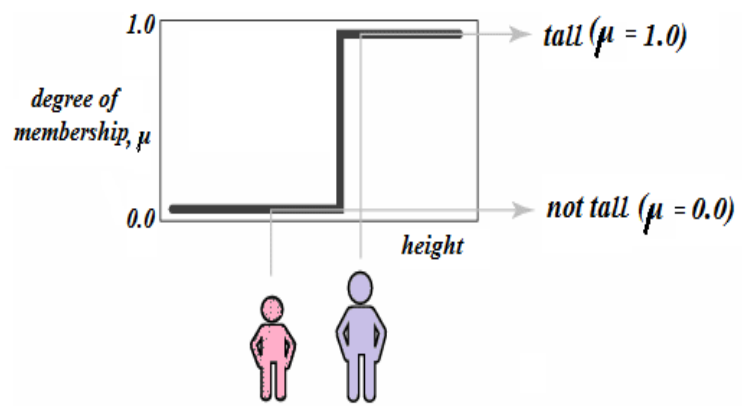

Fig 1: Sharp-edged (non-fuzzy) membership function for tall



Fig 2: Continuous-edged (fuzzy) membership function for tall

\section{FUZZY SET OPERATIONS}

Operation on fuzzy set is called fuzzy set operation. These operations are performed on fuzzy sets and fuzzy relations and are generalized form of crisp set operation. The standard fuzzy set operation is most widely used operation. There are the following three basic operations of fuzzy set [5], [10]:

- Fuzzy complements

- $\quad$ Fuzzy intersections

- $\quad$ Fuzzy unions

Let the fuzzy sets be $A$ and $B$, such that $A, B \in U$, $x$ is an element in the universe $\mathrm{U}$

4.1 Union: The union (combination) of two fuzzy sets $(A \cup B)$ is defined as-
$\varphi_{\mathrm{A} \cup \mathrm{B}}(x)=\max \left(\varphi_{\mathrm{A}}(x), \varphi_{\mathrm{B}}(x)\right)$

4.2 Intersection: The intersection of two fuzzy sets $(A \cap B)$ is defined as-

$\varphi_{\mathrm{A} \cap \mathrm{B}}(x)=\min \left(\varphi_{\mathrm{A}}(x), \varphi_{\mathrm{B}}(x)\right)$

4.3 Complement: The complement of the fuzzy sets is defined as-

For fuzzy set A: $\varphi_{\text {notA }}(x)=1-\left(\varphi_{\mathrm{A}}(x)\right)$

For fuzzy set B: $\varphi_{\text {notB }}(x)=1-\left(\varphi_{\mathrm{B}}(x)\right)$

\section{FUZZY LOGIC SYSTEM}

In 1972, Professor Toshire Terano organized the world's first working group on fuzzy logic systems. Fuzzy system is based on a logical system which is much closer to human thinking and natural language. It has good stability, fast response, less complexity and small overshoot than conventional systems. It has outstanding performance in dealing with complex, incorrectly defined, time varying and non linear systems. Figure 3 shows fuzzy logic system. Following are the basic required elements of fuzzy logic system [8], [9]:

\subsection{Fuzzifier}

The first most element of fuzzy logic system is fuzzifier. It is used in fuzzy logic space for mapping any crisp value into proper fuzzy value i.e. it is responsible for converting the crisp input into a fuzzy input which is used by the inference engine. It performs various functions:

- Measures input variables

- $\quad$ Scale mapping

- Fuzzification

\subsection{Fuzzy Rule Base}

Fuzzy rule base is the main part of fuzzy system which is made up of fuzzy rules. Fuzzy rules are in the form of IFTHEN statement. There are many other components in the system which helps in implementing the fuzzy rules in an appropriate manner. Fuzzy rule base vary from application to application.

\subsection{Inference Engine}

Inference engine is an artificial intelligence tool. It takes fuzzy inputs from the fuzzifier and processes the fuzzy rules on the fuzzy inputs.

\subsection{Defuzzifier}

Defuzzifier is responsible for converting the fuzzy value into the final crisp output value. According to the output variable membership function, defuzzification is performed.

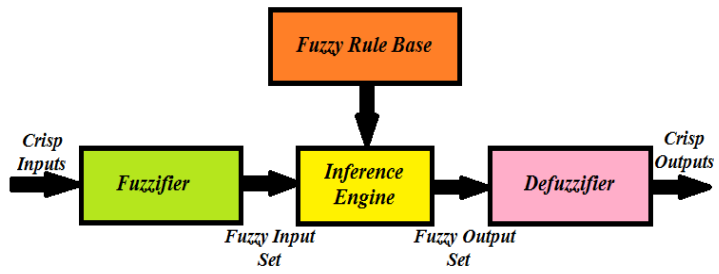

Fig 3: Fuzzy logic system 


\section{BENEFITS OF FUZZY LOGIC SYSTEM OVER CONVENTIONAL CONTROL SYSTEM}

Fuzzy logic enables a human being to easily interface with an automated system. In fuzzy logic system, fuzzy rules are defined that are in the form of natural language. Hence, fuzzy system has an ability to communicate directly with the user [1], [4].
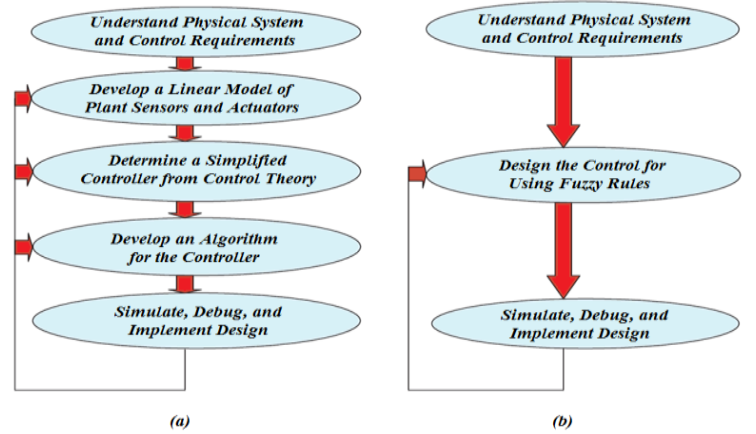

Fig 4: (a) Conventional control theory, (b) Logic theory

Figure 4 shows comparison between conventional control theory and fuzzy logic theory. The comparison points between them are given below:

- Conventional control system is difficult to understand as compared to fuzzy logic system.

- Conventional control system requires plant modeling and sensory information to produce control actions, while fuzzy logic controller needs only fuzzy rules for controlling actions.

- An algorithm for the conventional controller is difficult to develop, while fuzzy logic algorithms are simple and easy to generate.

- From the above points it is clear that the complexity of conventional control system is more as compared to fuzzy logic system.

\section{FUZZY INFERENCE SYSTEM}

Fuzzy inference system is a method in which the values are interprets in the input vector and according to the user defined rules; the values are assigning to the output vector. With the help of MATLAB fuzzy logic toolbox, rules set, membership functions can be defined and the behavior of the fuzzy inference system can be analyzed. Figure 5 shows the fuzzy inference system [1].

Fuzzy logic toolbox is a collection of graphical tools or command-line functions. With the help of these tools and functions, fuzzy inference system can create and edit within the MATLAB framework. In fuzzy logic toolbox, there are five elementary graphical user interface (GUI) tools for editing, building, and observing fuzzy interference system which are given below [6], [7], [11]:

- $\quad$ Fuzzy Inference System (FIS) Editor

- $\quad$ Membership Function Editor

- $\quad$ Rule Editor

- $\quad$ Rule Viewer

- $\quad$ Surface Viewer

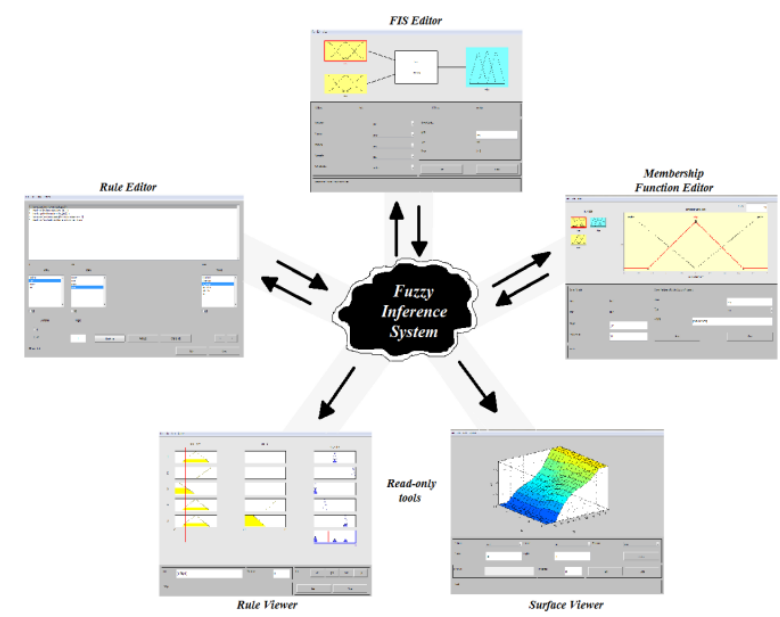

Fig 5: Fuzzy inference system

FIS editor is used to create a new fuzzy inference system from scratch. The FIS editor window is opened by typing 'fuzzy' on command window of the MATLAB. It displays general information about fuzzy inference system. The most common and easy methodology is the Mamdani's fuzzy inference method. Membership function editor is used to display and edit all of the membership functions concerned with all input and output variables for the whole fuzzy inference system. Rule editor makes the user to define or modify the rules for the fuzzy logic system. Rule viewer displays a roadmap of the entire fuzzy inference system. The related GUI tools can be open in the menu bar of rule viewer. After the completion of design, in the surface viewer window, a three dimensional curve (surface) of a system is obtained. It is possible to see, edit and save the designed surface.

\section{SIMULATIONS OF FUZZY LOGIC APPLICATIONS USING THE MATLAB}

Almost all the fuzzy logic applications used linguistic variables. Once understood the fuzzy logic, it is not difficult to implement fuzzy logic systems, and the results are usually more than satisfactory and quite surprising. Fuzzy logic found applications in signal processing, operations analysis, database management, data classifications, decision support systems, etc. Fuzzy logic is mainly use in applications that needs controlling. Here, some controlling and processing applications have been simulated using the fuzzy logic.

\subsection{Water level control}

In many residential areas, combined water tank systems are used. The functioning of these systems are accurate most of the time, but sometimes the system is unable to detect whether the water level reached the desired level properly or not and thus results in an overflow. Sometimes it is also unable to detect whether the inlet rate of water is proportional to the outlet rate or not and hence cause the water tank empty with much faster rate than it fills up. The solution for these problems is the construction of a new tank, but such schemes are expensive and complex too to extend the system networks across the wide geographical areas. Hence, the fuzzy logic controller is implemented in water tank systems for the controlling of level and rate of water. Figure 6 shows water tank and figure 7 shows water level control in a tank using fuzzy logic controller. This simulink model consists - inputs (level rate), water tank, valve, fuzzy logic controller, outputs 
(scopes). Figure 8 shows the control response of fuzzy logic controller using following five rules [1], [12], [13], [14]:

- If (level is okay) then (valve is no_change).

- If (level is positive) then (valve is open_fast).

- If (level is negative) then (valve is close_fast)

- If (level is okay) and (rate is positive) then (valve is close_slow).

- If (level is okay) and (rate is negative) then (valve is open_slow).

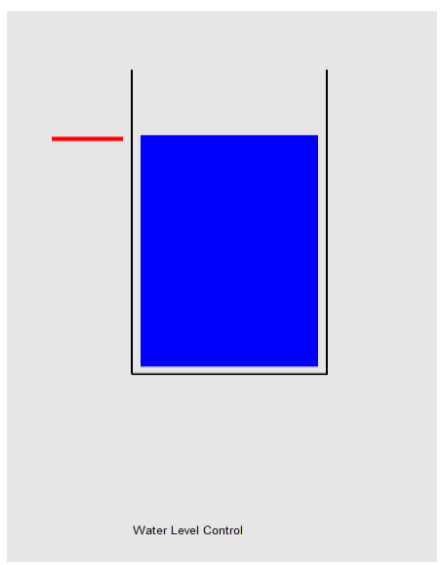

Fig 6: Water tank

Water Level Control in a Tank

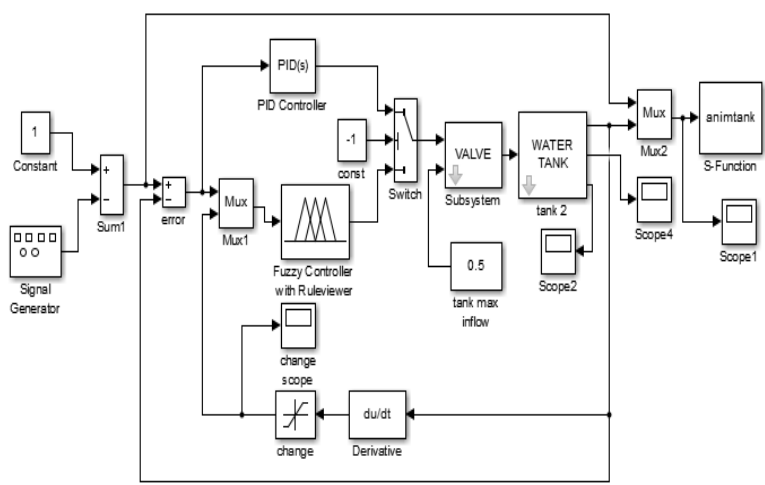

Fig 7: Water level control in a tank using fuzzy logic controller

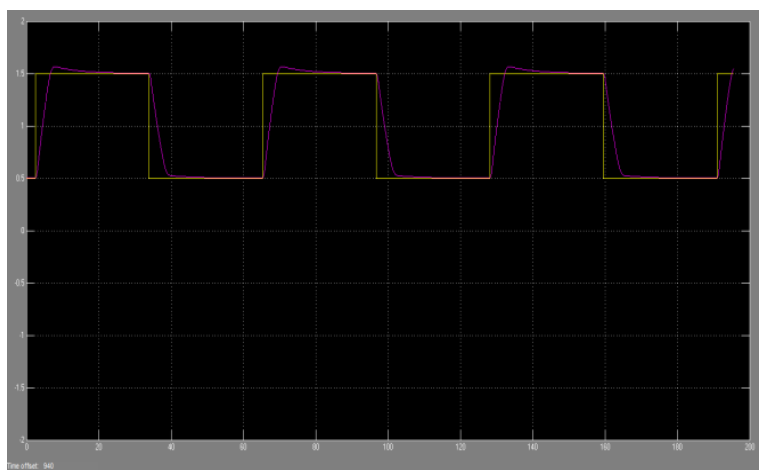

Fig 8: Control response of fuzzy logic controller

\subsection{Temperature control in a shower}

As the name implies, here the fuzzy logic controller is used in shower for controlling the outlet water temperature. In this, the different fuzzy rules are defined, according to which the hot water blends with cold water to provide constant, safe shower [1]

\section{Temperature Control in a Showe}

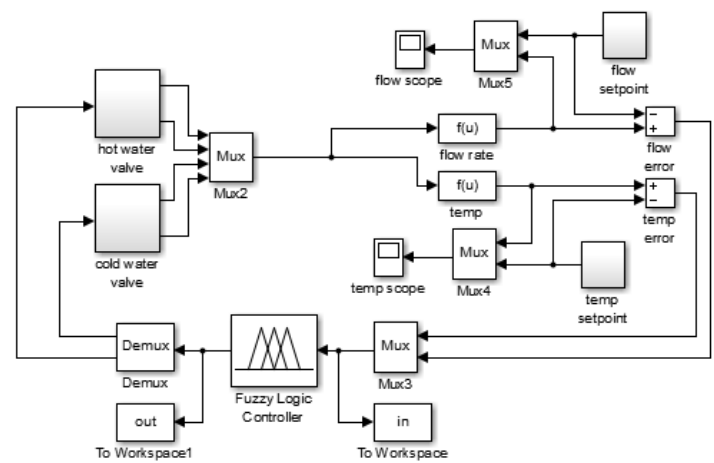

Fig 9: Temperature control in a shower using fuzzy logic controller

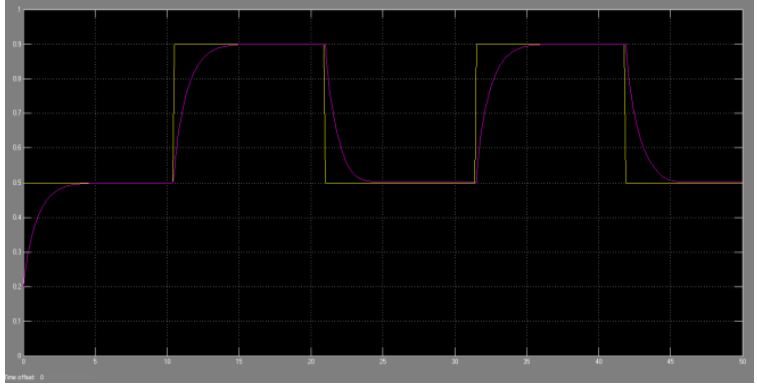

Fig 10: Flow scope

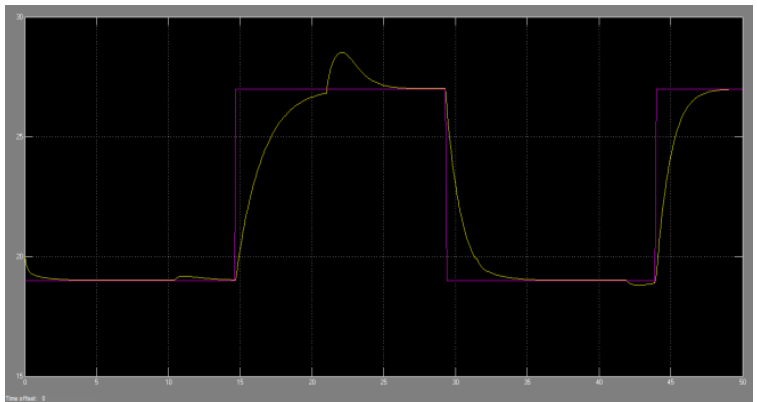

Fig 11: Temperature scope

Figure 9 shows the temperature control in shower using fuzzy logic controller. Here, the flow rate and temperature of the outlet water of shower is compared with the flow set point and temperature set point respectively for the calculation of flow error and temperature error. These two errors are act as inputs for the fuzzy logic controller. According to the input values and defined fuzzy rules, the fuzzy logic controller generates output which is used to control hot and cold water valve. Figure 10 and 11 shows flow scope and temperature scope respectively.

\subsection{Fuzzy logic image processing (edge detection)}

Edge detection is an image processing technique which is used for finding the objects boundaries within an image. In a 
digital image, it is used for identifying the points where the brightness of image changes sharply. These points are then consolidated into a set of curved line segments which termed as edges. The main aim of edge detection is to locate the pixels and detecting significant local changes in an image. Edge detection is an elemental tool in machine vision, computer vision and image processing, and generally used for segmentation and detection of image and for the extraction of data. Figure 12 shows input image in grayscale [15], [16], [17].

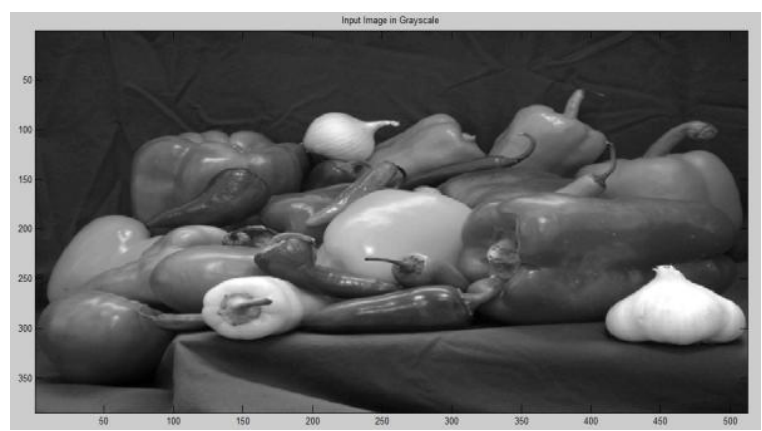

Fig 12: Input image in grayscale

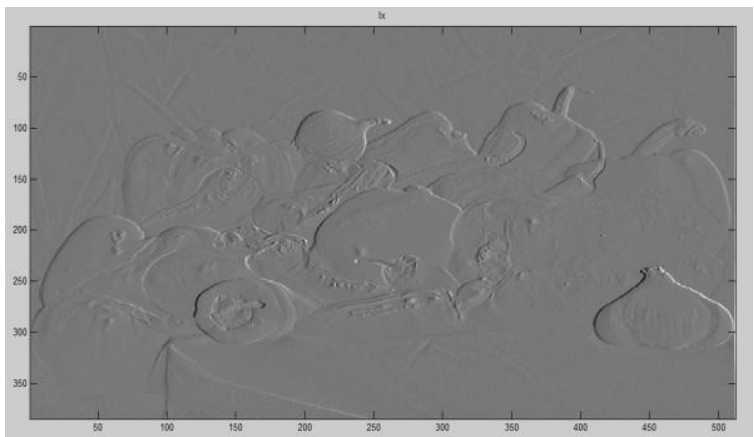

Fig 13: Gradient image in the $x$ direction

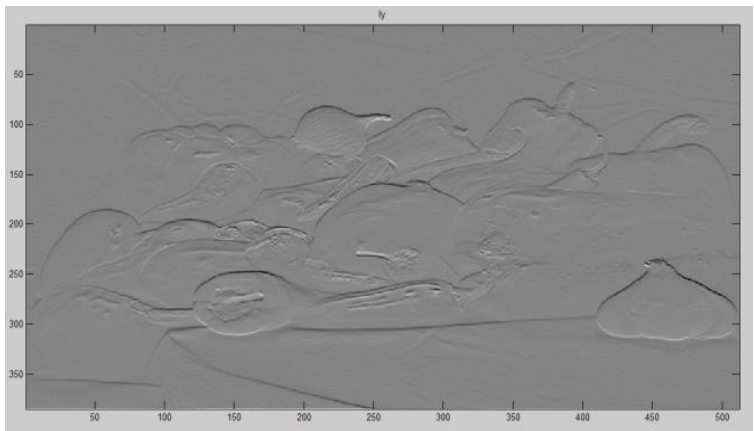

Fig 14: Gradient image in the $y$ direction

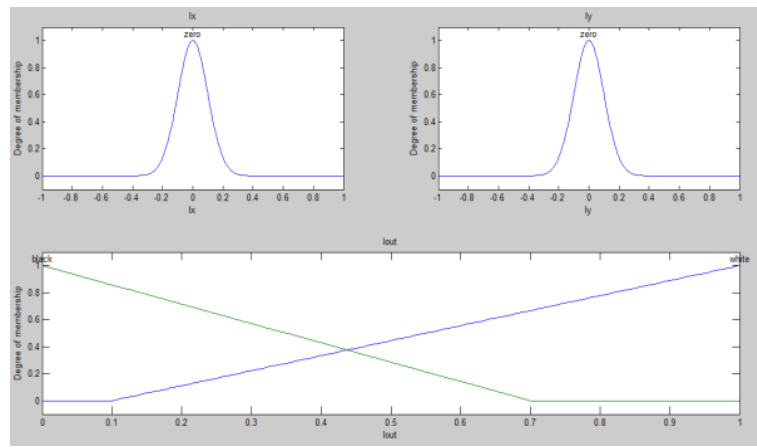

Fig 15: Membership functions of the inputs/outputs of edge FIS
Figure 13 shows the gradient image in the $\mathrm{x}$ direction which measures horizontal change in the brightness while figure 14 shows the gradient image in the y direction which measures vertical change in the brightness. These gradient images are specified as inputs of edge FIS and then zero-mean Gaussian function for each input is defined. After that a triangular membership function for an output of edge FIS is defined. Figure 15 shows the membership functions of the inputs/outputs of edge FIS [1].

An image gradient is nothing but a directional change in the brightness or intensity in an image. Gradient image is used for the extraction of information from an image. It can be created from the original image or grayscale of the original image simply by convolving with a filter.

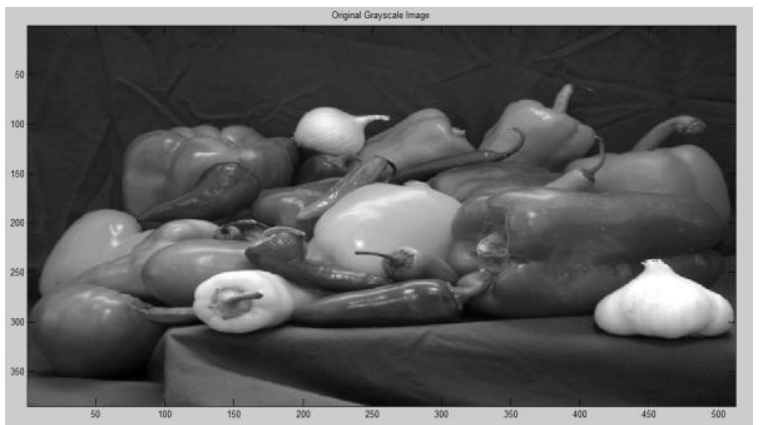

Fig 16: Original grayscale image

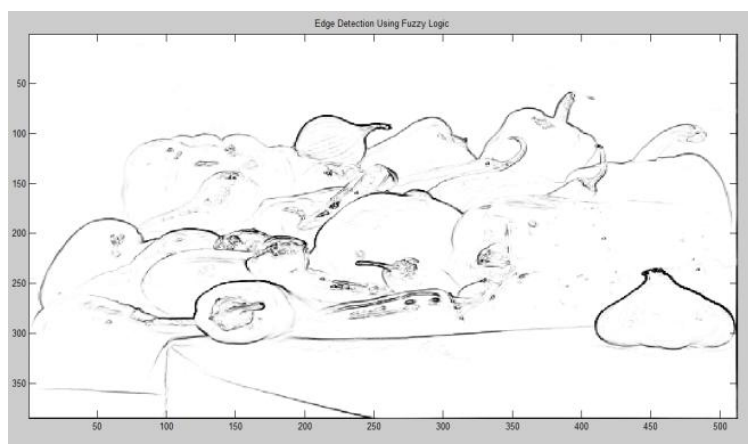

Fig 17: Edge detection of image using fuzzy logic

Figure 16 shows original grayscale image and figure 17 shows edge detection of image using fuzzy logic. The edge detection of an image is done by using FIS. In this the gradients of every pixel in the $\mathrm{x}$ and $\mathrm{y}$ direction are compared. The pixel belongs to an edge (black) if gradient for a pixel is not zero. The gradient is defined as zero by using Gaussian membership functions for the FIS inputs

\subsection{Adaptive Noise Cancellation}

An adaptive filter is essentially a digital filter used to eliminate an unwanted noise signal from the received signal in order to improve the signal to noise ratio (SNR). It has a self adjustment characteristics i.e. it has an ability to adjust its own coefficient automatically. Figure 18 shows adaptive noise canceller model [18], [19]. 


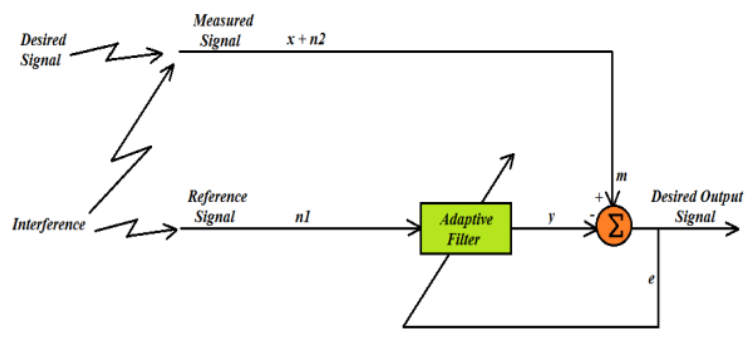

Fig 18: Adaptive noise canceller (ANC) model

Here, an adaptive filter has two inputs- measured signal (m) and reference signal (n1). The measured signal contains two signals- a desired signal (x) and an interference signal (n2) i.e. $\mathrm{m}=\mathrm{x}+\mathrm{n} 2$. The interference signal $(\mathrm{n} 2)$ is uncorrelated with the original signal (x) but correlated with reference signal (n1). The reference signal is filtered by an adaptive filter and subtracted from the measured signal in order to regain the desired signal. The error signal (e) is feedback to the adaptive filter for the adjustment of its coefficient [1], [20].

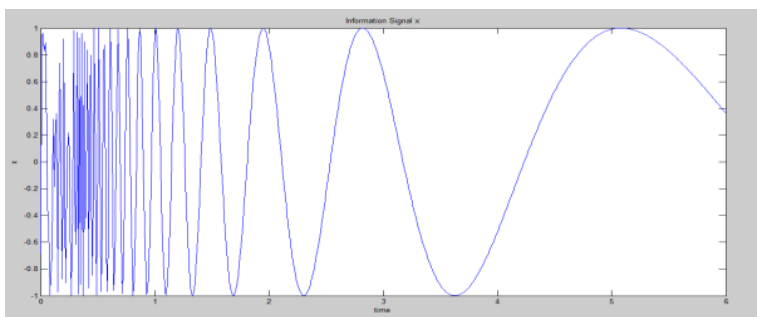

Fig 19: Original information signal $x$.

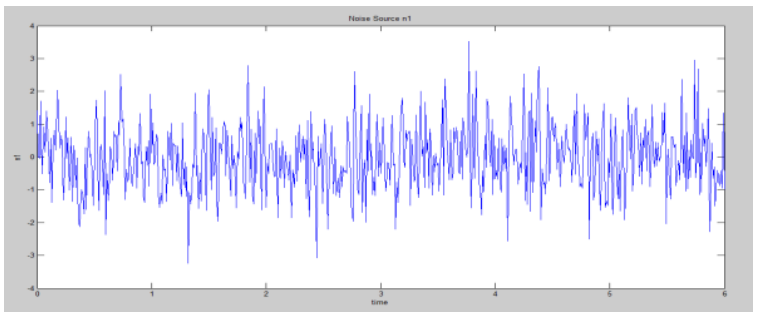

Fig: 20 Noise source n1

Figure 19 shows the original information signal and figure 20 shows the noise source (n1) which generates an interference signal (n2) for the signal (x). Signal $\mathrm{n} 1$ act as a reference signal which correlates in some way with $\mathrm{n} 2$. The interference signal (n2) is assumed to be generated via an unknown nonlinear equation which is given below:

$$
n 2(k)=\frac{4 * \sin (n 1(k)) * n 1(k-1)}{1+n 1(k-1)^{2}}
$$

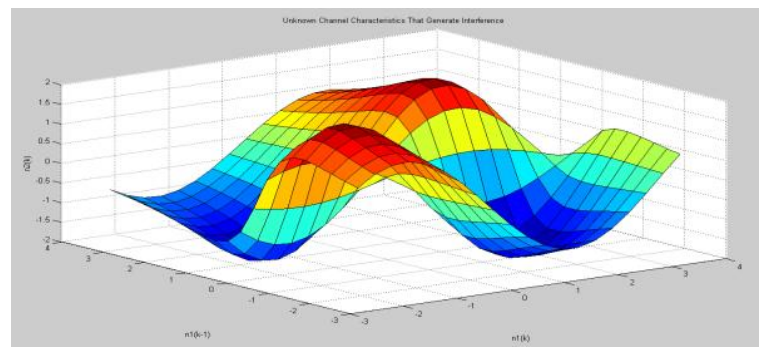

Fig 21: Surface for the non-linear characteristics of the channel that generates interference signal (n2)

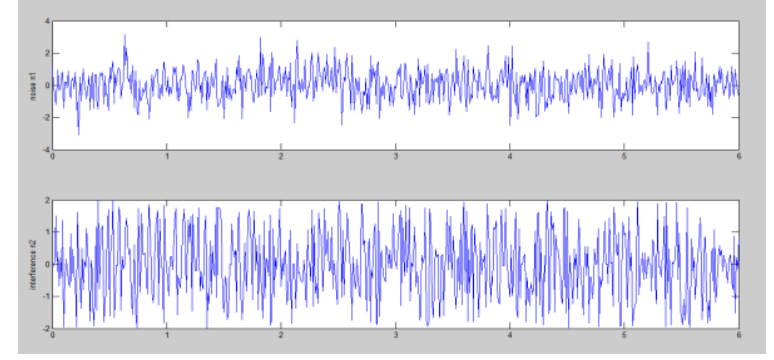

Fig 22: Noise signal (n1) and interference signal (n2)

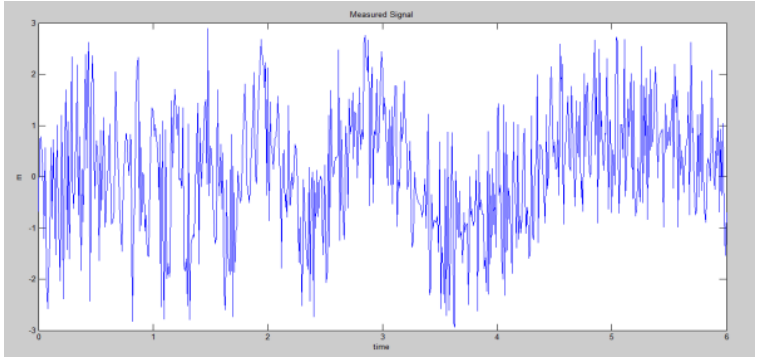

Fig 23: Measured signal (m)

Figure 21 shows surface for the non-linear characteristics of the channel that generates interference signal (n2). In figure 22 , the noise signal (n1) and interference signal (n2) are shown together. Figure 23 shows the measured signal (m) which is sum of the original information signal ( $\mathrm{x}$ ) and the interference signal (n2) i.e. $m=x+n 2$.

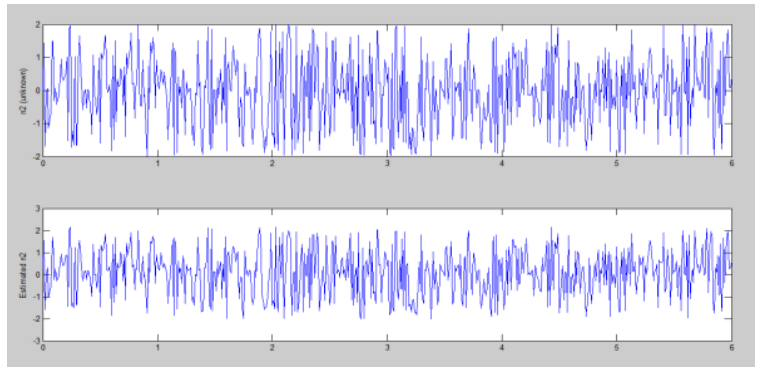

Fig 24: Unknown interference signal (n2) and estimated signal (n2)

With the help of ANFIS (Adaptive Neuro-Fuzzy Inference System), the non-linear relationship between $\mathrm{n} 1$ and $\mathrm{n} 2$ can be identified. The ANFIS function is used to construct a fuzzy inference system (FIS) whose parameters of membership function are adjusted using either in combination with a least squares type of method or a back-propagation algorithm alone. Figure 24 shows an unknown interference signal (n2) and an estimated signal (n2). The estimated signal is subtracted from the measured signal to get the estimated information signal (x).

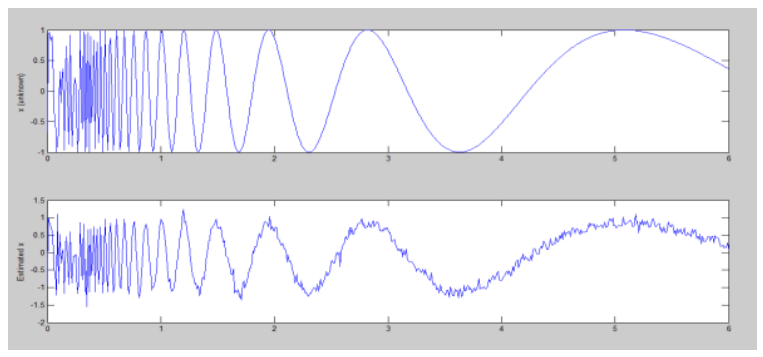

Fig 25: Original information signal and estimated information signal 
Figure 25 shows the original information signal and the estimated information signal which is obtained by using ANFIS. Both the signals are highly correlated and hence, SNR is improved.

\section{CONCLUSION}

This paper presents a detailed description of fuzzy logic which clears that fuzzy logic is an alternative way to represent linguistic and subjective attributes of the real world. In order to improve the efficiency and simplicity of the design process, fuzzy logic can be applied to various control systems and other applications. The comparison points between fuzzy logic system and conventional system which is described in this article concludes that fuzzy logic systems are simple, easy to understand and have less complexity than other controlling systems. This paper makes the readers to have a broad understanding of fuzzy logic and its advantages, fuzzy sets, fuzzy set operations, fuzzy logic system, fuzzy inference system (FIS) and various application based on fuzzy logic for further purpose of investigation and aware the engineers and educators how to enhance the applications of fuzzy logic and renovate the conventional control systems by using fuzzy rules. The various applications of fuzzy logic which is simulated using the MATLAB prove that fuzzy logic systems do a fairly good job than other controlling systems. Meanwhile, this paper can also trigger the further investigations related to the integration of fuzzy logic controller with soft computing techniques.

\section{REFERENCES}

[1] Fuzzy Logic Toolbox, for use with MATLAB, "The MathWorks", user's guide, version 2.

[2] Kiran Pal,Surendra Tyagi, "Selection of Candidate by Political Parties Using Fuzzy Logic", International Conference of Advance Research and Innovation (ICARI - 2004).

[3] Pedro Albertos and Antonio Sala, "Fuzzy Logic Controllers. Advantages and Disadvantages", September 14, 1998.

[4] Fuzzy logic, https://en.m.wikipedia.org/wiki/Fuzzy_logic

[5] Fuzzy set operations, https://en.m.wikipedia.org/wiki/Fuzzy_set_operations

[6] Anita Pati, V.K. Singh, K.C. Mishra, "Filtering Noise on two dimensional image Using Fuzzy Logic Technique, International Journal of Scientific \& Engineering Research, Volume 2, Issue 3, March-2011, ISSN 22295518.

[7] Siddharth Saxena and Rajeev Kumar Singh, "A Novel Approach of Image Restoration on Segmentation and Fuzzy Clustering", International Journal of Signal Processing, Image Processing and Pattern Recognition, Vol.7, No.4 (2014),pp.255-264.

[8] Nivedita Chakraborty and Minakshi Deb Barma, department of Electrical Engineering, NIT Agartala, Tripura, India, "Modelling of Stand -Alone Wind Energy Conversion System using Fuzzy Logic Controller", International Journal of Innovative Research in Electrical, Electronics, Instrumentation and Control Engineering, Vol. 2, Issue 1, January 2014, ISSN (Online) 2321 - 2004, ISSN (Print) 2321 - 5526.
[9] Fuzzy logic, http://reference.wolfram.com/applications/fuzzylogic/Ma nual/3.html

[10] Fuzzy set operations, http://www.maplesoft.com/applications/view.aspx?SID= $14171 \&$ view $=$ html.

[11] Inma P.Cabrera, Pablo Cordero, and Manuel OjedaAciego, "Fuzzy Logic, Soft Computing, and Applications".

[12] Disha, Mr. Pawan Kumar Pandey, Rajeev Chugh, "Simulation of Water Level Conrol in a Tank Using Fuzzy Logic, IOSR Journal of Electrical and Electronics Engineering (IOSRJEEE), ISSN: 2278-1676 Volume 2, Issue 3 (Sep-Oct. 2012), PP 09-12.

[13] Harshdeep Singh (109ME0422), "Design of Water Level Controller Using Fuzzy Logic System", National Institute of Technology Rourkela.

[14] Bourdillon O. Omijeh, M. Ehikhamenle, Elechi Promise, " Simulated Design of Water Level Control System", Computer Engineering and Intelligent Systems, Vol 6, No.1, 2015.

[15] Tarun Mahashwari, Amit Asthana, "Image Enhancement Using Fuzzy Technique" International Journal of Research Review in Engineering Science \& Technology, Volume-2, Issue-2, June-2013.

[16] Er. Mandeep Singh Sandhu, Er. Vikram Matneja, Er. Nishi, "Edge Detection by Using Rule Based Fuzzy Clasifier", International Journal of Computer Science and Information Technologies, Vol 2 (5), 2011, 24342439.

[17] Abdallah A. Alshennawy, and Ayman A. Aly, "Edge Detection in Digital Images Using Fuzzy Logic Technique", International Journal of Computer, Electrical, Automation, Control and Information Engineering, Vol:3, No:3, 2009.

[18] Reena Rani, Dushyant Kumar (Asst. Prof), Narinder Singh, " Design of Adaptive Noise Canceller Using RLS Filter a Review", International Journal of Advanced Research in Computer Science and Software Engineering, Volume 2, Issue 11, November 2012.

[19] MARVIN R. SAMBUR, member, IEEE, "Adaptive Noise Canceling for Speech Signals", IEEE TRANSACTIONS ON ACOUSTICS, SPEECH, AND SIGNAL PROCESSING, VOL, ASSP-26, NO.5, OCTOBER 1978.

[20] Sayed. A. Hadei, Student Member IEEE and M. Iotfizad, "A Family of Adaptive Filter Algorithms in Noise Cancellation for Speech Enhancement", International Journal of Computer and Electrical Engineering, Vol. 2, No. 2, April 2010, 1793-8163. 